\title{
Introduction to Anterior Cranial Fossa and Craniofacial Region Lesions
}

The source of growth for the majority of tumors of the anterior cranial fossa (ACF) and craniofacial region (CFR) is a variety of structures and tissues in the specified area. These include: dura mater, olfactory nerves, mucosa of the upper nasal cavity and paranasal sinuses, orbital structures, tooth and the bones themselves. Neoplasms of this region of the head can be broadly classified from either epithelial or mesenchymal origin. Epithelial tumors may arise from mucosa (papillomas, squamous cell carcinomas, and adenocarcinomas), neuroendocrine tissue (sinonasal neuroendocrine carcinomas), minor salivary gland (from benign to malignant ones), and olfactory mucosa (olfactory neuroblastoma). Most often primary malignant tumors of this area are the squamous cell carcinoma, adenocarcinoma, and rarely occur melanoma and lymphoma. Metastases to this area are quite rare too. Among non-neoplastic lesions, inflammatory changes in the sinuses of the skull, different types of polyps, which, growing into the ACF region, can mimic neoplasms, are often encountered in radiological practice. Imaging of most ACF and CFR lesions is primarily aimed at determining the expansion of the lesion rather than determining its histological type, whether the tumor grows into ACF from the skull base, whether the brain substance is involved in the pathological process. What brain and vascular structures are involved in the affected area? In addition, there is a number of other issues in the evaluation of lesion expansion at the skull base, which radiologists have to resolve. For example, if the lesion spreads into the orbit. Tumors can often grow through the lamina papyracea and raise the periosteum of the orbit, not spreading into the orbital fat through this fibrous membrane. Extraconal fat in relation to the medial rectus muscle is a very important reference point: if the fat is intact and protrusion of the tumor into the orbital fat has a smooth edge, the lesion is limited by the periosteum.
If the tumor grows into the paranasal sinuses, a low MR-signal from the air is replaced by the signal representing the tumor. This is an important diagnostic symptom. However, inflammation or obstruction of the sinus cavity by inflammatory exudate also changes the signal in the sinus. Tumors themselves often cause a sinus cavity obstruction. In this case, both the tumor and inflammatory obstructive changes may be detected in the same sinus. The use of additional MR-sequences (inflammatory obstruction of the sinus has a high MR-signal on T2- and T2*-weighted images more often than tumors) or intravenous administration of contrast agents (most of skull base tumors enhance, while secretion into the sinus doesn't) is sometimes required in order to specify these issues.

The accuracy of topical lesion evaluation significantly increases when different MR-pulse sequences and modalities are used and the study is performed on multiple planes. In particular, a combination of orthogonal planes with oblique reformations better demonstrates expansion of the tumor into the skull base and CFR structures. The roof of the orbit and the cribriform plate are better visualized on the coronal images. Transition from the base of the ACF to the frontal bone wall is a smooth curve, along which neither axial nor coronal plane forms perpendicular slices, thus, cortical bone lines may be blurred. Sagittal images appear to be more informative in such cases. Routine diagnostic protocol of MR-studies of ACF and CFR has to include MR-sequences with fat signal suppression (both $\mathrm{T} 2$ - and $\mathrm{T} 1$-weighted sequences), while post-contrast scanning is best to be obtained using spin echo T1-weighted sequences with fat signal suppression or new MR-technologies with Dixon method of fat saturation (T1-IDEAL (GE)) for better discrimination of the enhanced lesion from surrounding tissues (Curin and Cunnane 2009). 